Single crystals of a compositionally complexed equiatomic FeNiCrCo alloy were successfully grown to investigate the fundamental deformation mechanisms.

Single crystal of FeNiCrCo alloy

\title{
$20 \mathrm{~mm}$
}

Laue diffraction pattern

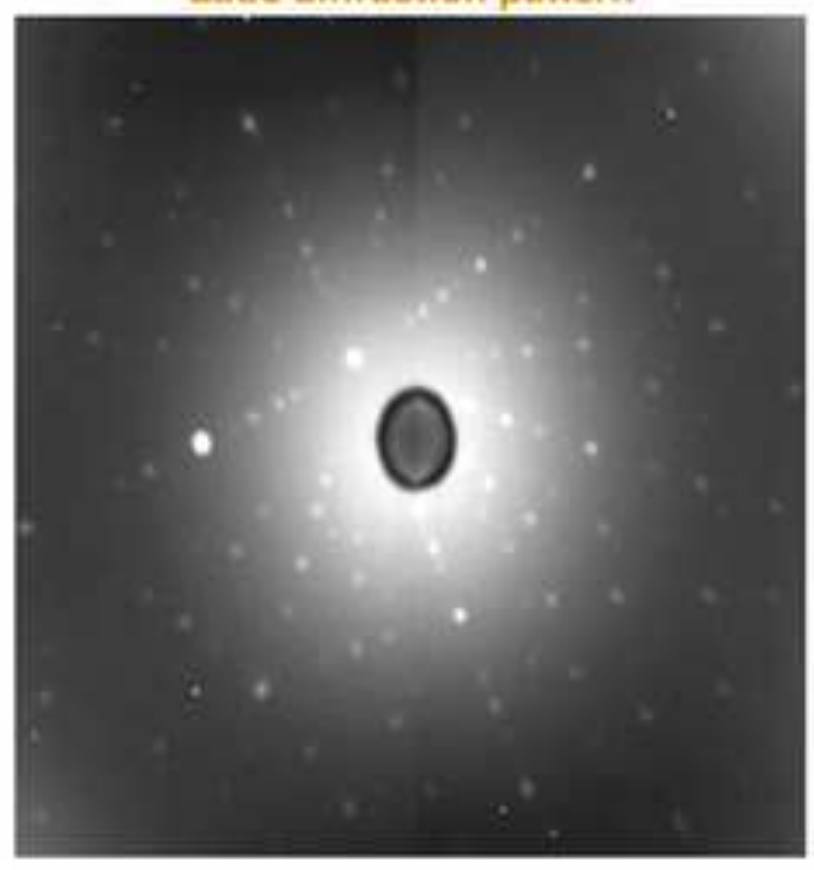

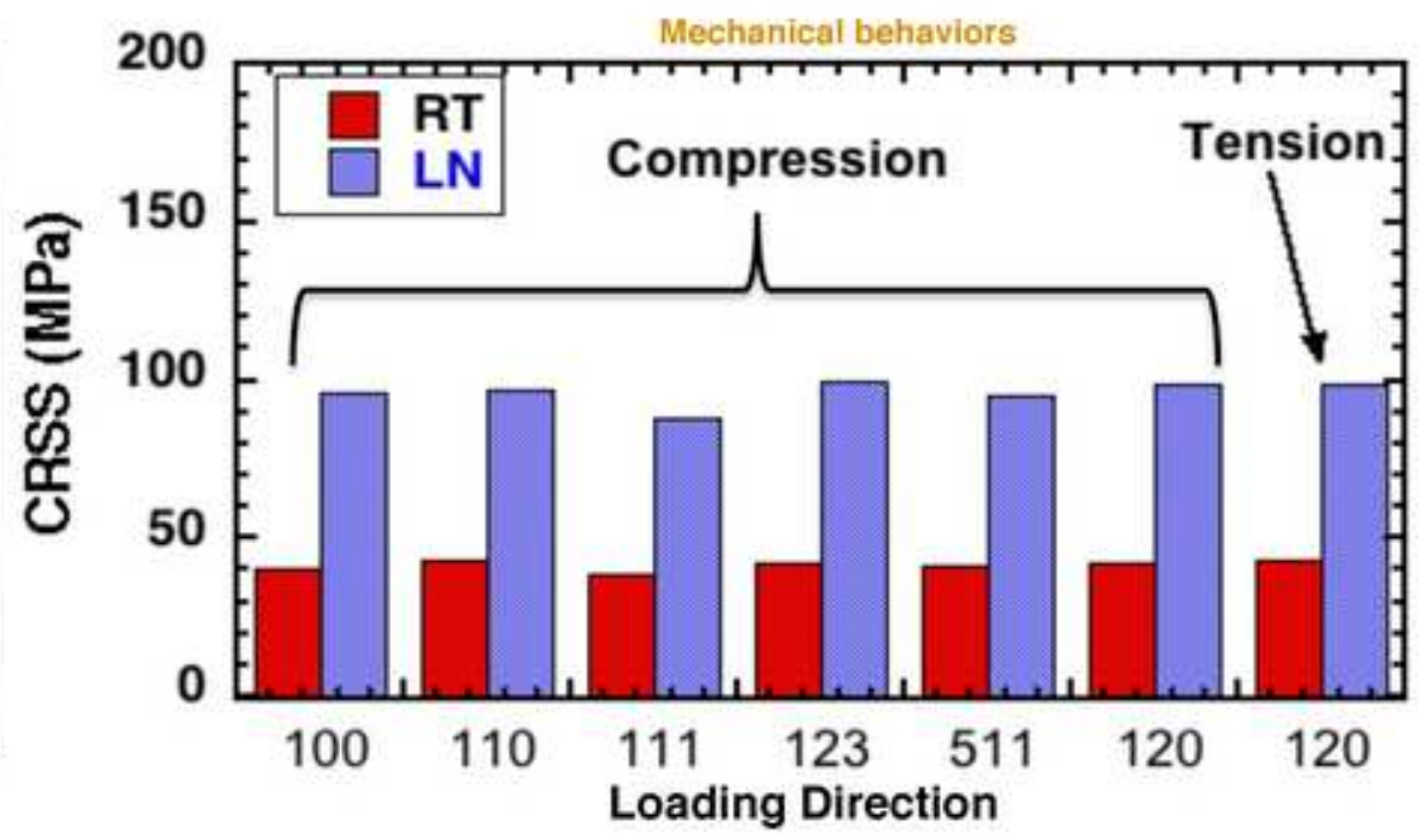




\title{
Single crystal plastic behavior of a single-phase, face-center-cubic- structured, equiatomic FeNiCrCo alloy *
}

\author{
Z. Wu ${ }^{\mathrm{a}, \mathrm{b}}$, Y.F. Gao ${ }^{\mathrm{a}, \mathrm{b}}, \mathrm{H} . \mathrm{Bei}^{\mathrm{a} * *}$ \\ a Materials Science and Technology Division, Oak Ridge National Laboratory, Oak \\ Ridge, TN 37831 USA \\ b Materials Science and Engineering Department, University of Tennessee, Knoxville, \\ TN 37996 USA
}

\begin{abstract}
To understand the fundamental deformation mechanisms of compositionally complex alloys, single crystals of a multi-component equiatomic $\mathrm{FeNiCoCr}$ alloy with face-centercubic (FCC) structure were grown for mechanical studies. Similar to typical FCC pure metals, slip trace analyses indicate that dislocation slips take place on (111) planes along [1̄ㅣㄹ directions. The critical resolved shear stress (CRSS) obeys the Schmid law at both 77 and $293 \mathrm{~K}$, and tension-compression asymmetry is not observed. Although this material slips in a normal FCC manner both at 293 and $77 \mathrm{~K}$, the strong temperature dependence of the CRSS is abnormal in comparison to the typical FCC metals.
\end{abstract}

Keywords: Compositionally complex alloys; Single crystal plasticity; Temperature dependence; Slip trace analysis; high entropy alloys

\footnotetext{
* This manuscript has been authored by UT-Battelle, LLC under Contract No. DE-AC05-00OR22725 with the U.S. Department of Energy. The United States Government retains and the publisher, by accepting the article for publication, acknowledges that the United States Government retains a non-exclusive, paid-up, irrevocable, world-wide license to publish or reproduce the published form of this manuscript, or allow others to do so, for United States Government purposes. The Department of Energy will provide public access to these results of federally sponsored (http://energy.gov/downloads/doe-public-access-plan).

** Corresponding author: Beih@ornl.gov (Email); (865)5767196 (phone) (865) 5747659
} 
Recent theoretical and experimental studies have demonstrated an alloy development concept to combine five or more metallic elements in equal atomic proportions to form a single phase, random solid solution alloy with a simple crystal structure, such as face-centered cubic (FCC) and body-centered cubic (BCC) [1-7], which was usually called high entropy alloys (HEAs). The reason that such compositionally complex alloy can remain structurally-simple might be that the entropy of mixing is large enough to overcome the enthalpy associated with compound or multi-phase formation [6, 7]. Although the number of "actual" high entropy alloys with equiatomic compositions (as truly stable single-phased solid solution) is limited [5], those that do exist often exhibit interesting mechanical properties, including high strength and ductility $[8,9]$. For example, contrary to most traditional materials in which an inverse temperaturedependence of strength and ductility is usually observed, the FCC FeNiCrCoMn HEA shows simultaneous increases in both strength and ductility with decreasing test temperature (e.g., from 293 to $77 \mathrm{~K}$ ) [8, 9]. The formation of nanoscaled mechanical twinning during deformation were observed at low temperature of $77 \mathrm{~K}$, which can act as additional deformation modes to potentially enhance the strain hardening, uniform elongation and fracture toughness. Moreover, such behaviors are not only observed in the 5-element FeNiCrCoMn alloys, but also in some low-number-element alloys, e.g., 4element equiatomic FeNiCrCo and even 3-element equiatomic NiCoCr alloys [10-12].

Despite the above progress, previous experimental observations are based on mechanical testing of polycrtsalline materials, which cannot answer some of the fundamental questions related to single crystal plasticity [13]. In polycrystalline materials, the stress state of individual grains becomes very complicated and individual grains are 
no longer subjected to a uniaxial stress state even when the specimen is deformed uniaxially. Because the slip systems in neighboring grains are oriented differently, the need for an individual grain to accommodate the slip fields in the neighboring grains will make the slip systems operating near a grain boundary frequently different from those elsewhere. Grain boundaries can act as obstacles to dislocation movement, and such an interaction is affected by many factors such as temperature and grain size. All of these make it difficult to mechanistically understand the deformation mechanisms of the alloy, particularly the questions on the slip behavior in these materials. For example, is strength different in tension and compression? Does the critical resolved shear stresses obey or violate the Schmid law? To this end, single crystals with their sizes large enough to conduct mechanical testing are required [13]. Here, we report that single crystals of the FeNiCoCr alloy are successfully grown and their mechanical properties and microstructures are explored to reveal fundamental mechanical behavior of the equiatomic alloy. We select 4-element $\mathrm{FeNiCoCr}$ alloy as a model alloy for two considerations. First, the mechanical behavior of this alloy is almost identical to the 5elmente FeNiCrCoMn alloy [12], and thus is representative in compositionally complex alloys. Second, the extensive Mn evaporation during single crystal growth can be avoided for this material, making our single crystal growth facility feasible. Mechanical studies of these single crystals will lay solid foundations for future experimental and theoretical studies.

Single crystals of equiatomic FeNiCrCo alloys are grown in an optical floating zone furnace from polycrystalline rods, which were produced by arc melting pure $\mathrm{Fe}, \mathrm{Ni}$, Co and $\mathrm{Cr}$ followed by drop casting into a copper mold under Ar atmosphere. Details of 
materials preparation and single crystal growth can be found elsewhere $[14,15]$. The quality and orientation of the crystals were examined by the backscatter Laue diffraction and metallography. The growth direction of the single crystal is around [012]. The asgrown crystals were re-oriented in a goniometer by checking its backscatter Laue diffraction pattern and specimens were cut for mechanical testing. The compressive samples have a dimension of $3 \times 3 \times 6 \mathrm{~mm}^{3}$ and the tensile samples are of dog bone shape with a gauge dimension of $1.2 \times 2 \times 10 \mathrm{~mm}^{3}$. The surfaces of the samples are carefully metallographically polished in order to reveal the slip trace pattern after the tests. Uniaxial compression and tension were conducted in a screw driven Instron loading frame with initial strain rate of $5 \times 10^{-4} \mathrm{~s}^{-1}$. Microindentation was also performed on (001), (110) and (111) surfaces using a spherical sapphire indenter with a radius of $\sim 100 \mu \mathrm{m}$ to generate surface slip traces that could be imaged in a microscope. A JEOL JCM-5000 microscope was used to characterize its microstructures before and after mechanical testing. Atom probe tomography characterizations were performed in voltage-pulsed mode with a CAMECA Instruments Inc. LEAP 4000X HR local electrode atom probe $[16,17]$ to reveal atomic distribution of the $\mathrm{Fe}, \mathrm{Ni}, \mathrm{Cr}$ and $\mathrm{Co}$ elements and nanoscaled microstructures in the single crystal.

Figure 1 shows the three-dimensional $\mathrm{Fe}, \mathrm{Ni}, \mathrm{Cr}$ and $\mathrm{Co}$ atomic maps in the single crystal of equiatomic FeNiCrCo alloy. No precipitates or nanoscaled clusters were observed, indicating random distribution of the existing alloying elements. Therefore, the current equiatomic alloy should be treated as a "pure" material with mysterious "effective medium" of stoichiometric compounds with fixed atomic ration instead of traditional solid solution with "solvent" and "solute" species, indicating that the traditional 
strengthen mechanism and deformation behavior, which was developed for dilute FCC solid solution alloy, might not apply for this type materials.

Figure 2 shows the slip traces of the equiatomic FeNiCoCr alloy for (001), (011) and (111) oriented surfaces under spherical microindentation. Typically, FCC crystals have four unique slip planes, each of which has three slip directions, resulting in 12 independent slip systems as shown in Figure $2 a$. Slip steps will appear along the line direction that corresponds to the intersection of the slip plane and the sample surface since they are the result of dislocations gliding through the free surface. For a perfect (001) type crystal, both the (111) and (111) slip planes will intersect the (001) surface along the $[1 \overline{1} 0]$ directions, and both the (111) and (11̄1) slip planes will intersect the (001) surface along the [110] direction. Thus there will be two sets of possible line directions for the slip steps. These orientations are very symmetrical and each of the four $\{111\}$ planes has the same orientation with respect to the axis of indentation. Therefore, the resolved shear stress should be equal for all four slip-planes and equal amounts of slip would be expected for each slip plane, leading to the prediction of a square-like pattern.

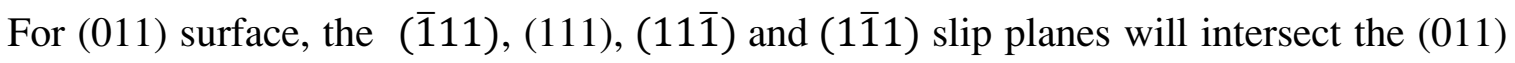
along the $[01 \overline{1}],[0 \overline{1} 1],[2 \overline{1} 1]$ and $[\overline{2} \overline{1} 1]$ directions, respectively. The cross angle between $[\overline{2} 1 \overline{1}]$ and $[2 \overline{1} 1]$ is $70.52^{\circ}$. They will have a cross angle of $54.73^{\circ}$ with $[01 \overline{1}]$ and $[0 \overline{1} 1]$, respectively, making a slip pattern as shown in Figure $2 b$. For a perfect (111) surface, there will be three sets of possible directions for the slip steps. That is, (111) slip

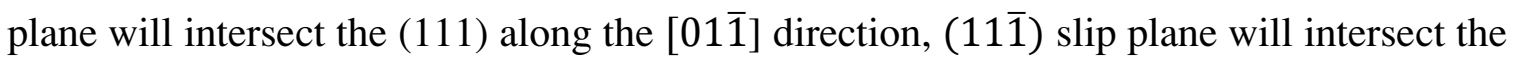

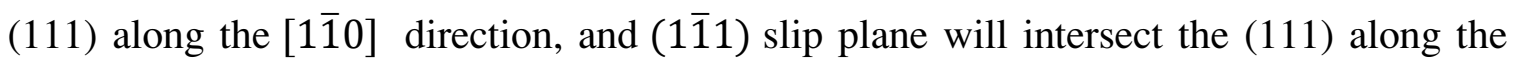
[101] direction. The cross-angles between any two is either 60 or $120^{\circ}$, making a 
triangle-like pattern. It can be seen that the slip step patterns formed on the surface surrounding the impression of the FeNiCoCr alloy (Fig. 2) are well consistent with the predicted ones. This suggests that, similar to traditional FCC metals, dislocations in the multi-component equiatomic $\mathrm{FeNiCoCr}$ alloy also slip in the $\{111\}$ planes along the $<110>$ directions.

The stress-strain curves for the equiatomic $\mathrm{FeNiCoCr}$ alloy with various orientation are shown in Figure 3. The two crystals shown in the figure have their long axes oriented to [100] and [110] respectively, and the orientation of the side surfaces are also indicated in the inset. For both cases, following yielding is a two-stage hardening process, which is characterized by a low and linear rate of hardening (Stage 1) followed by a region of more significant hardening (Stage 2). The presence of multiple stages of hardening has been shown in numerous FCC metals with high purity, such as $\mathrm{Al}, \mathrm{Ag}, \mathrm{Ni}$ and $\mathrm{Cu}$, and believed to be a typical FCC characteristics for crystals with orientations having limited primary slip systems. This is the case for the current [110] oriented crystal in which only two types of slip systems (A and D) are observed to be activated (Fig. $2 d$ ). For high-purity FCC metals in $<100>$ orientations, the easy glide region (Stage 1) is usually absent due to the presence and simultaneous activation of multiple slip systems. However, Stage 1 is rather found in the investigated FeNiCoCr [100] crystals. One possible reason could be that only limited primary slip systems are indeed activated. This can be supported by the representative image depicting the slip trace on the (110) side surfaces in which typical features of $\{111\}<110>$ type slip lines are observed. These lines correspond well to the plane $\mathrm{C}$ (i.e. to the system $\mathrm{C} 4$ and/or C6), plane A (i.e. A4 and/or A5) as shown in Figure 2a. The $\mathrm{C}$ type slip lines are the predominant ones and cross the 
face entirely, remaining well parallel to each other. The A type slip lines are sparsely distributed. It should be noted that these traces intersect without deviation; this tend to prove that the two slip systems acting in this case have the same slip direction [18], which would designate the systems A4 and $\mathrm{C} 4$. We also note that for single crystal plasticity with noticeable Stage 1 deformation behavior, a small misoreintation from symmetric directions such as [100] can actually lead to the activation of only one slip system, as shown by numerical simulations in [19]. Further investigations will be needed to have a complete understanding the suppression of certain slip systems. The extended stage 1 at $77 \mathrm{~K}$ compared to that at $293 \mathrm{~K}$ as shown in Fig. $3 a$ and $b$ is also a typical FCC feature and believed to be associated with the rise in the critical shear stress for both primary and secondary slip [20].

In addition to the [100]- and [110]-oriented crystals, the stress-strain curves and slip patterns of a few other crystals with various orientations are also characterized. In all crystals, no other slip systems than the $\{111\}<100>$ type are observed. Similar typical deformation mechanism for FCC materials has already been reported for the equiatomic FeNiCoCrMn alloy in which dislocation motion was found to be associated with glide of $1 / 2<110>$ dislocations on $\{111\}$ planes [8]. The yield strengths of these differently oriented crystals are collected in Table I, included in which are also the possible primary slip systems with the highest Schmid factor. The critical resolved shear stresses calculated based on the Schmid law for crystal with different orientations falls in the same range. All of these could be regarded as a verification of the Schmid law of critical resolved shear stress. Another feature typical for FCC materials, i.e., the tension/compression symmetry, is also observed [20]. The same values of yield strength 
are obtained under tension and compression for the (012) crystal as shown in Figure 4.

Results in Figs. 2, $3 \& 4$ show that although the FeNiCrCo alloy behaves in a typical FCC manner in slip behavior, its CRSS was strongly temperature dependent, which is not typical in pure FCC metals, e.g., Ni. Yield stress is a combination of the frictional stress plus the various types of incremental strengthening contributions, such as precipitate hardening $\left(\Delta \sigma_{\mathrm{ppt}}\right)$, initial dislocation density $\left(\Delta \sigma_{\mathrm{\rho i}}\right)$, and grain boundary (HallPetch) strengthening $\left(\Delta \sigma_{\mathrm{gb}}\right)$. For a carefully prepared single crystal, $\Delta \sigma_{\rho \mathrm{i}}$ and $\Delta \sigma_{\mathrm{gb}}$ can be eliminated right away due to the rather low initial dislocation density and thus its weak contribution to the overall strength, as well as the absence of grain boundaries. Because no cluster and precipitants were observed, $\Delta \sigma_{\mathrm{ppt}}$ can also be removed. The solid solution hardening $\left(\Delta \sigma_{\mathrm{ss}}\right)$ in such equiatomic alloys will be reflected in the frictional stress $\left(\sigma_{\mathrm{fr}}\right)$, which is the intrinsic lattice resistance to dislocation motion. Therefore, yield stresses measured in such single crystals reflects the intrinsic internal frictional stress in the materials.

The CRSS is almost doubled when the temperature decreases from 293 to $77 \mathrm{~K}$. This temperature dependence is believed to be caused by the effects of thermal energy on the amount of short-range barrier which needs thermal activation to overcome [11]. It is well known that in BCC metals, the Peierls barrier can act as a strong short-range obstacle for moving dislocations due to the non-planar core structure of screw dislocation, causing the thermal activation of dislocation glide [21-23]. In contrast, the Peierls barrier is negligibly small in FCC metals, leading to very weak temperature dependence of yield strength [24-27]. As shown in Figs. 3 and 4, the temperature dependent yield stresses (or CRSS) measured in FeNiCrCo single crystals reflects the internal frictional stress in the 
materials, although this materials slip (dislocation glide) behave in normal FCC manner both in 293 and 77K. Because of the random distribution of alloying elements (Fig. 1), it is suggested that the dislocation will be subjected to a nonuniform stress field due to the lattice, valency, and modulus mismatch among these atoms. The thermally activated process of the dislocation gliding will be thus governed by some rate-determining process that involves a volume much larger than the kink-pair process in BCC metals. To this end, it is anticipated that the Peierls barrier in our FeNiCrCo alloys is lower than that in BCC but much higher than that in typical FCC metals. In pure and dilute FCC metals, the temperature dependence of yield strength exhibits three different regions: low temperature thermal part, athermal intermittent part and high temperature thermal part. [20] If strong short-range barriers were presented, which might be caused by the shorter distance between atoms of different kinds in multi-component equiatomic alloys, the low temperature thermal regions could extend to higher temperatures, Moreover, due to the slower diffusion, the transition temperatures between the athermal and high temperature thermal region (diffusion-controlled) could also be higher in multi-component alloys as observed in the polycrystalline FeNiCoCrMn alloys. [8] Whether the strong temperature dependence of the yield strength in $\mathrm{FeNiCrCo}$ single crystals extends to higher temperature is subjected to future investigation.

In summary, single crystal $\mathrm{FeNiCoCr}$ equiatomic alloy was successfully grown and its mechanical properties were systematically studied. In general, this multicomponent FCC-structured alloy deforms like typical FCC materials. Dislocations tend to glide on the $\{111\}$ planes along the $<110>$ directions, making $\{111\}<110>$ primary slip systems. The CRSS obeys the Schmid law and tension-compression asymmetry is not 
observed. Although this material slips as in a normal FCC manner both in 293 and 77K, CRSS is found to be strongly temperature dependent as a typical BCC metal; and by eliminating the possibilities of influences from grain boundary and precipitates, this was believed to arise from the variation of intrinsic frictional stress with temperature.

\section{Acknowledgments}

This work was supported by the Department of Energy, Office of Science, Basic Energy Sciences, Materials Sciences and Engineering Division. The authors thank Dr. M. K. Miller in Oak Ridge National Laboratory for the help in APT conducted at the Center for Nanophase Materials Sciences, which is a DOE Office of Science User Facility.

\section{References}

[1] B. Cantor, I.T.H. Chang, P. Knight, et al.; Mater Sci Eng A 375-377 (2004): 213.

[2] M.S. Lucas, G.B. Wilks, L. Mauger, et al.; Appl Phys Lett 100 (2012): 251907.

[3] O.N. Senkov, G.B. Wilks, J.M. Scott, et al.; Intermetallics 19 (2011): 698.

[4] O.N. Senkov, J. M. Scott, S.V Senkova, et al.; J Alloys Compd 509(2011): 6043.

[5] H. Bei; US Patent Appl. Pub No. 2013/0108502 A1.

[6] S. Guo, C.T. Liu; Prog Nat Sci 21 (2011): 433.

[7] J.W. Yeh, S. K. Chen, S.J. Lin, et al.; Adv Eng Mater 6 (2004): 299.

[8] F. Otto, A. Dlouhy, CH. Somsen, et al.; Acta Mater 61 (2013): 5743.

[9] B. Gludovatz, A. Hohenwarter, D. Catoor, et al.; Science 345 (2014): 1153.

[10] Z. Wu, H. Bei, F. Otto, et al.; Intermetallics 46 (2014): 131.

[11] Z. Wu, H. Bei; Mater Sci Eng A 640 (2015): 217.

[12] Z. Wu, H. Bei, G. M. Pharr, et al.; Acta Mater 81 (2014):428.

[13] H. Y. Yasuda, K. Shigeno, T. Nagase, Scr. Mat. in press (2015)doi:10.1016/j.scriptamat.2015.06.022

[14] Y.Z. Xia, H. Bei, Y.F. Gao YF et al.; Mater Sci Eng A 611 (2014): 177.

[15] H. Bei, E. P. George; Acta, Mater. 53 (2004): 67.

[16] M.K. Miller, R.G. Forbes; Atom Probe Tomography: The Local Electrode Atom Probe, Springer, New York, NY, 2014. 
[17] D.J. Larson, T.J. Prosna, R.M. Ulfig, et al.; Local Electrode Atom Probe Tomography, Springer, New York, NY, 2013.

[18] P. Viliechaise, J. Mendez, P. Violan; Acta Metall Mater 39 (1991): 1683.

[19] F. Zhang, A.F. Bower, R.K. Mishra, et al.; Int. J. Plasticity 25 (2009): 49.

[20] R.W.K. Honeycombe; The Plastic Deformation of Metals. Edward Arnold LTD 1968.

[21] V. Vitek; Dislocations and Properties of Real Materials. The Institute of Metals, London, 1985. p30.

[22] M.M. Hutchison; Philos Mag A 8 (1963): 121.

[23] J.H. Bethtold; Acta Metall (3) 1955: 249.

[24] J.R. Low; Behavior of Metals at Low Temperatures. ASM. 1952, p48.

[25] T.H. Wille, C.H. Schwink; Acta Metall (34) 1986: 1059.

[26] H. Suzuki, in: P.O. Kettunen, T.K. Lepisto, M.E. Lehtonen (Eds.), Strength of Metals and Alloys (ICSMA 8), Pergamon Press, Oxford, 1988; 2: 573.

[27] H. Traub, H. Neuhauser, C.H. Schwink; Acta Metall (25) 1977: 437. 


\section{Figure captions:}

Figure 1 Atom probe tomography (APT) shows atomic distribution of the $\mathrm{Fe}, \mathrm{Ni}, \mathrm{Cr}$, Co elements in the single crystal of FeNiCrCo alloy.

Figure 2 (a) The 12 possible independent slip systems for FCC materials; (b) predicted and (c) observed slip trace pattern for (100), (011) and (111)-oriented single crystals under spherical indentations at 293 and $77 \mathrm{~K}$, respectively.

Figure 3 The representative stress-strain curves for the equiatomic FeNiCoCr single crystals with their loading axes parallel to (a) [100], and (b) [110] crystallographic orientation at 293 and $77 \mathrm{~K}$, respectively. SEM images show the corresponding slip lines on the shaded surfaces in (a) and (b).

Figure 4 The represented 293 and $77 \mathrm{~K}$ stress-strain curves for (012)-oriented single crystals tested in (a) compression, and (b) tension. SEM image show the corresponding slip lines in the (2 $\overline{6} 3$ ) surface of the tensile sample. 


\section{Tables}

Table I. Summary of possibly activated slip systems, maximum Schmid factor, $0.2 \%$ offset yield stresses and critical resolved shear stress of equiatomic FeNiCrCo single crystals.

\begin{tabular}{|c|c|c|c|c|c|c|}
\hline \multirow{2}{*}{$\begin{array}{r}\text { Loading } \\
\text { direction }\end{array}$} & \multirow{2}{*}{$\begin{array}{c}\text { Possible activated } \\
\text { slip systems }\end{array}$} & \multirow{2}{*}{$\begin{array}{c}\text { Schmid } \\
\text { factor }\end{array}$} & \multicolumn{2}{|c|}{ Yield Stresses (MPa) } & \multicolumn{2}{|c|}{ CRSS (MPa) } \\
\hline & & & $293 \mathrm{~K}$ & $77 \mathrm{~K}$ & $293 \mathrm{~K}$ & $77 \mathrm{~K}$ \\
\hline \multicolumn{7}{|l|}{ Compression } \\
\hline 100 & $\mathrm{~A} 4, \mathrm{~A} 5, \mathrm{~B} 3, \mathrm{~B} 6, \mathrm{C} 4, \mathrm{C} 6, \mathrm{D} 3, \mathrm{D} 5$ & 0.41 & 105 & 238 & 41 & 96 \\
\hline 110 & $\mathrm{~A} 2, \mathrm{~A} 4, \mathrm{D} 1, \mathrm{D} 3$ & 0.408 & 105 & 238 & 43 & 97 \\
\hline 111 & B3,B6,C1,C6,D1,D3 & 0.272 & 145 & 328 & 39 & 89 \\
\hline 123 & B3 & 0.467 & 91 & 205 & 42 & 96 \\
\hline 511 & $\mathrm{~A} 4, \mathrm{~A} 5$ & 0.454 & 91 & 214 & 41 & 97 \\
\hline 012 & A4, B3 & 0.49 & 85 & 201 & 42 & 98 \\
\hline \multicolumn{7}{|l|}{ Tension } \\
\hline 012 & $\mathrm{~A} 4, \mathrm{~B} 3$ & 0.49 & 86 & 203 & 42 & 99 \\
\hline
\end{tabular}



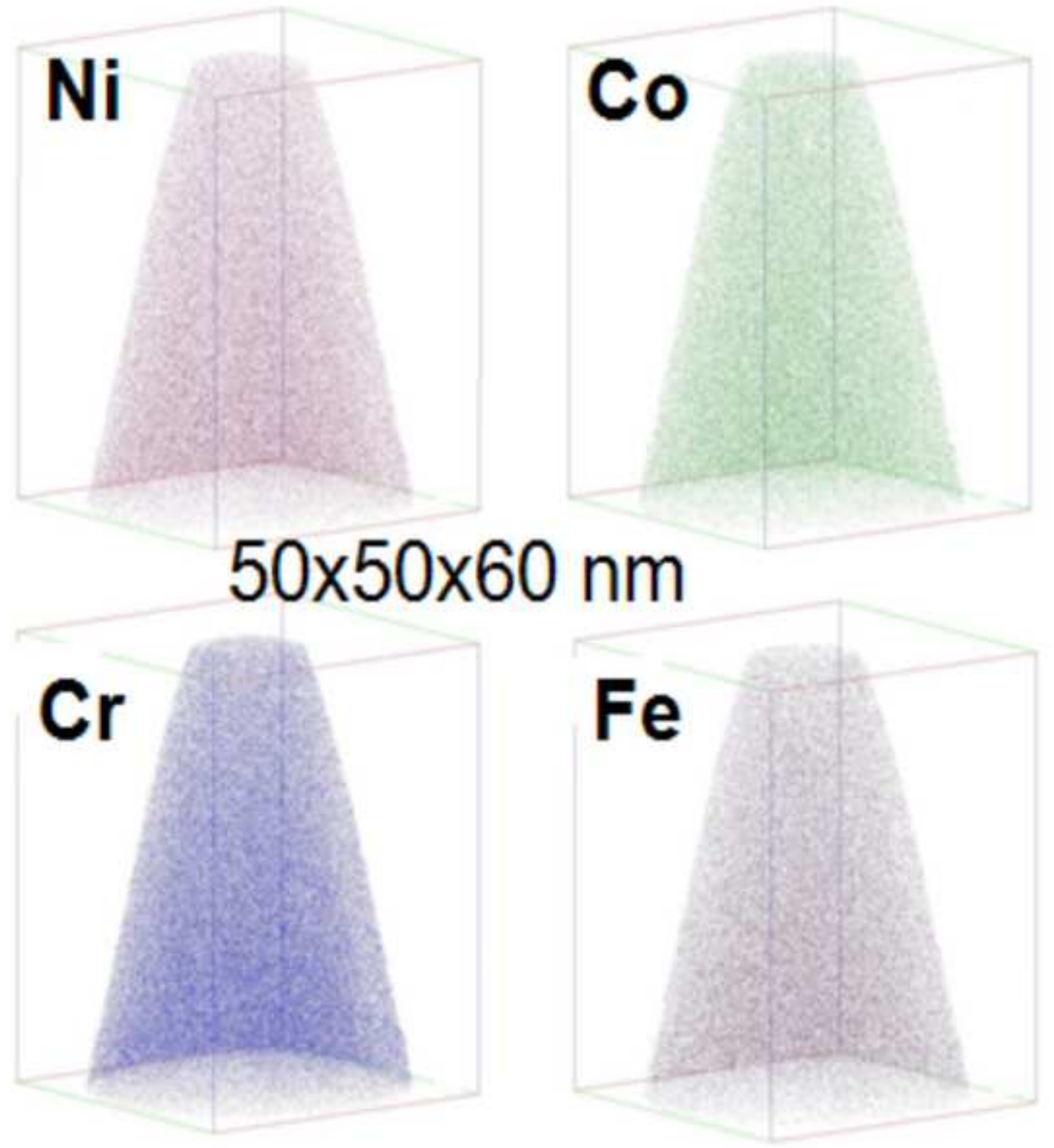

Figure 1

\section{$50 \times 50 \times 60 \mathrm{~nm}$}

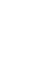


(a)

Slip planes

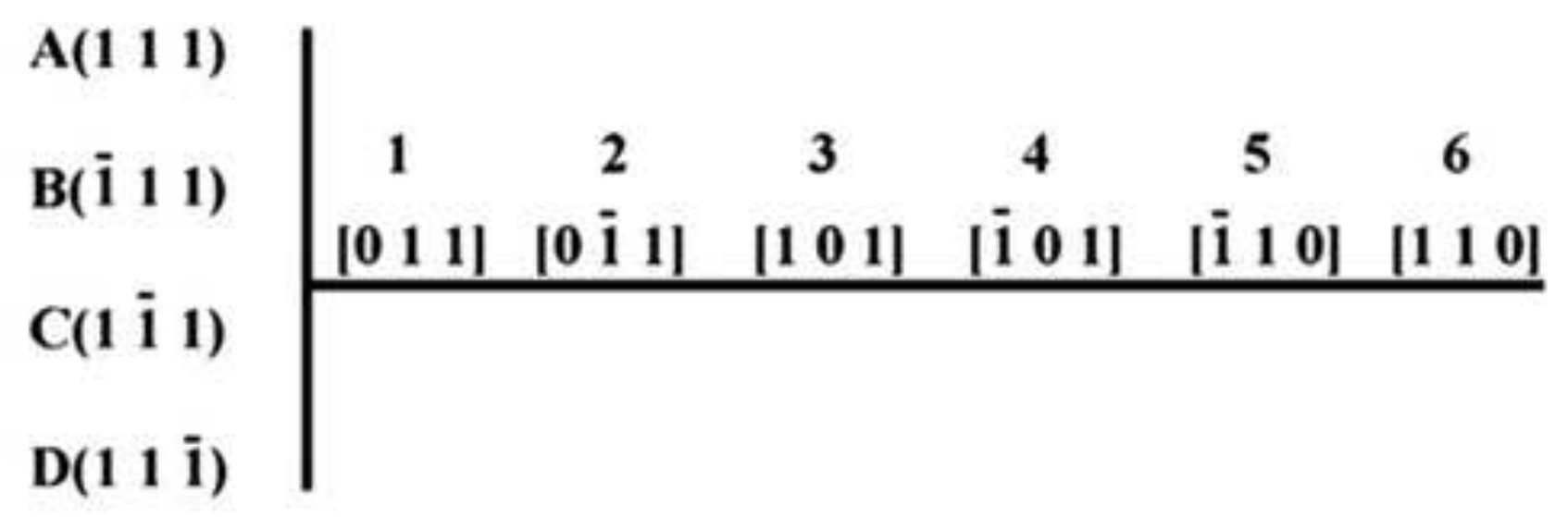

(b)

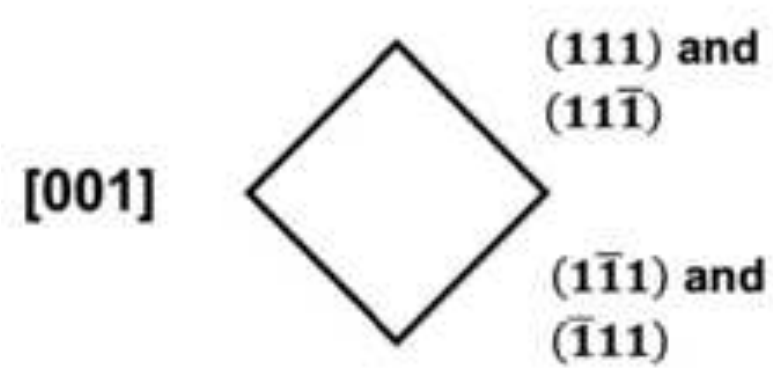

[011]

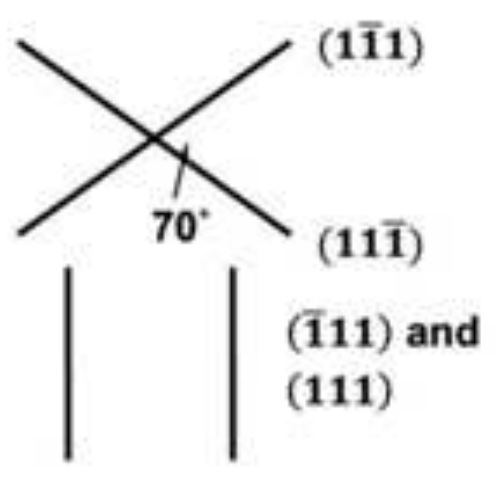

[111]

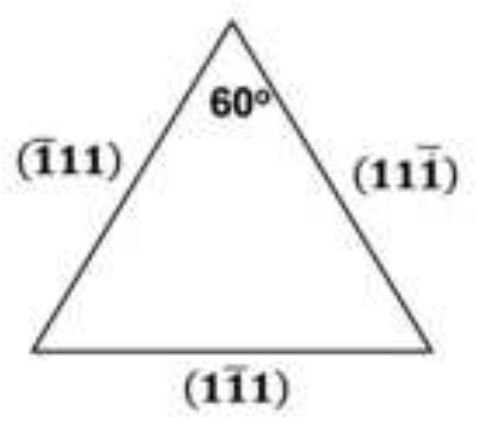

(c)
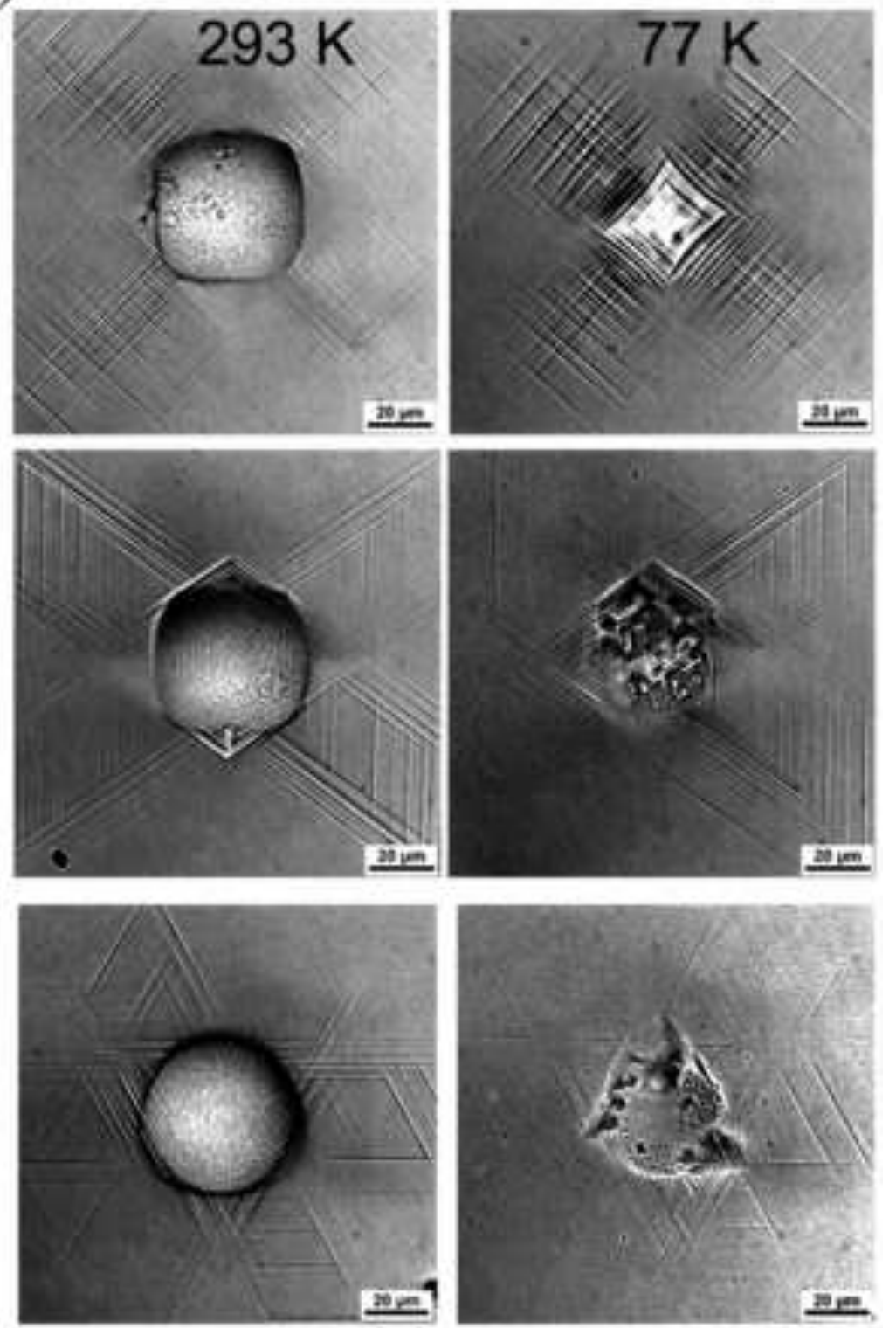
(a)

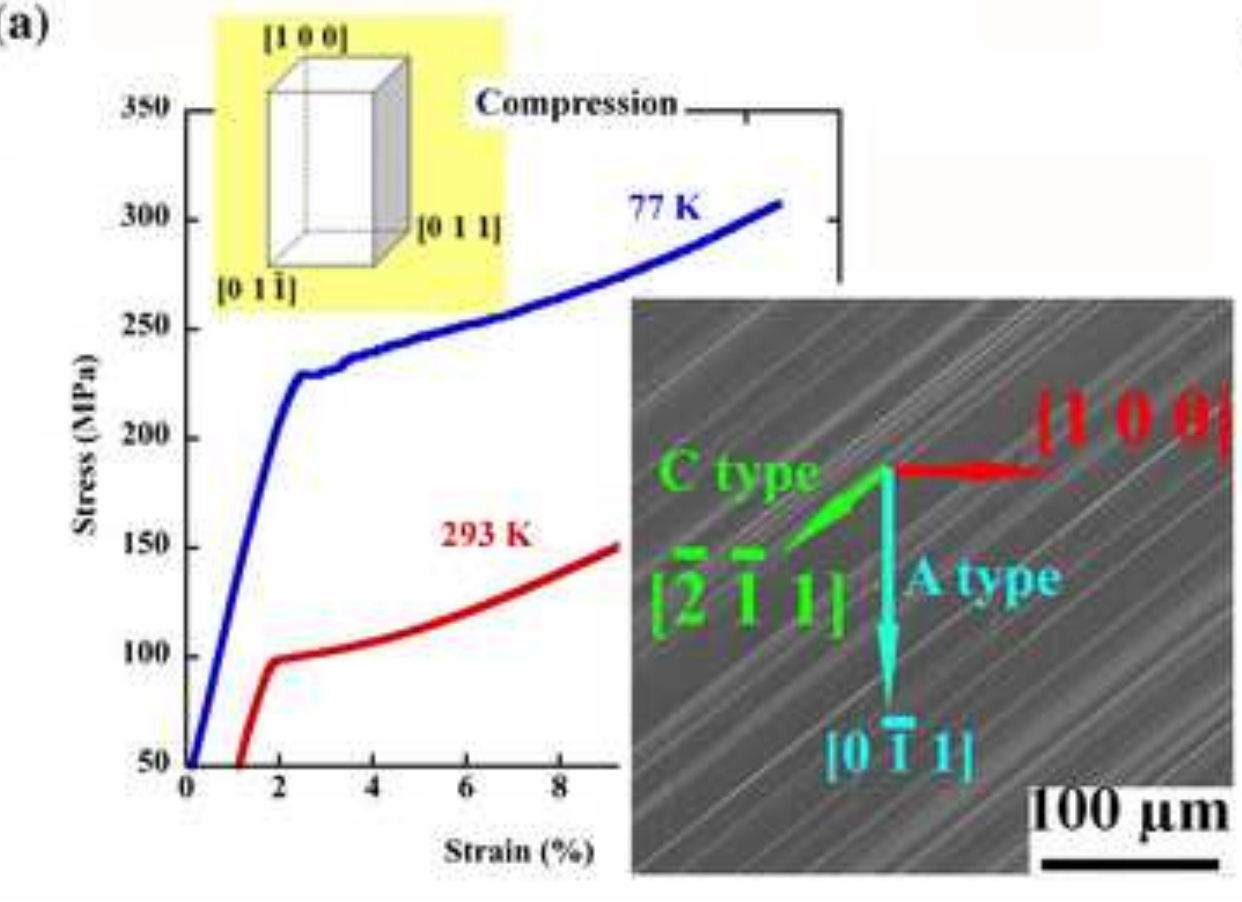

(b)

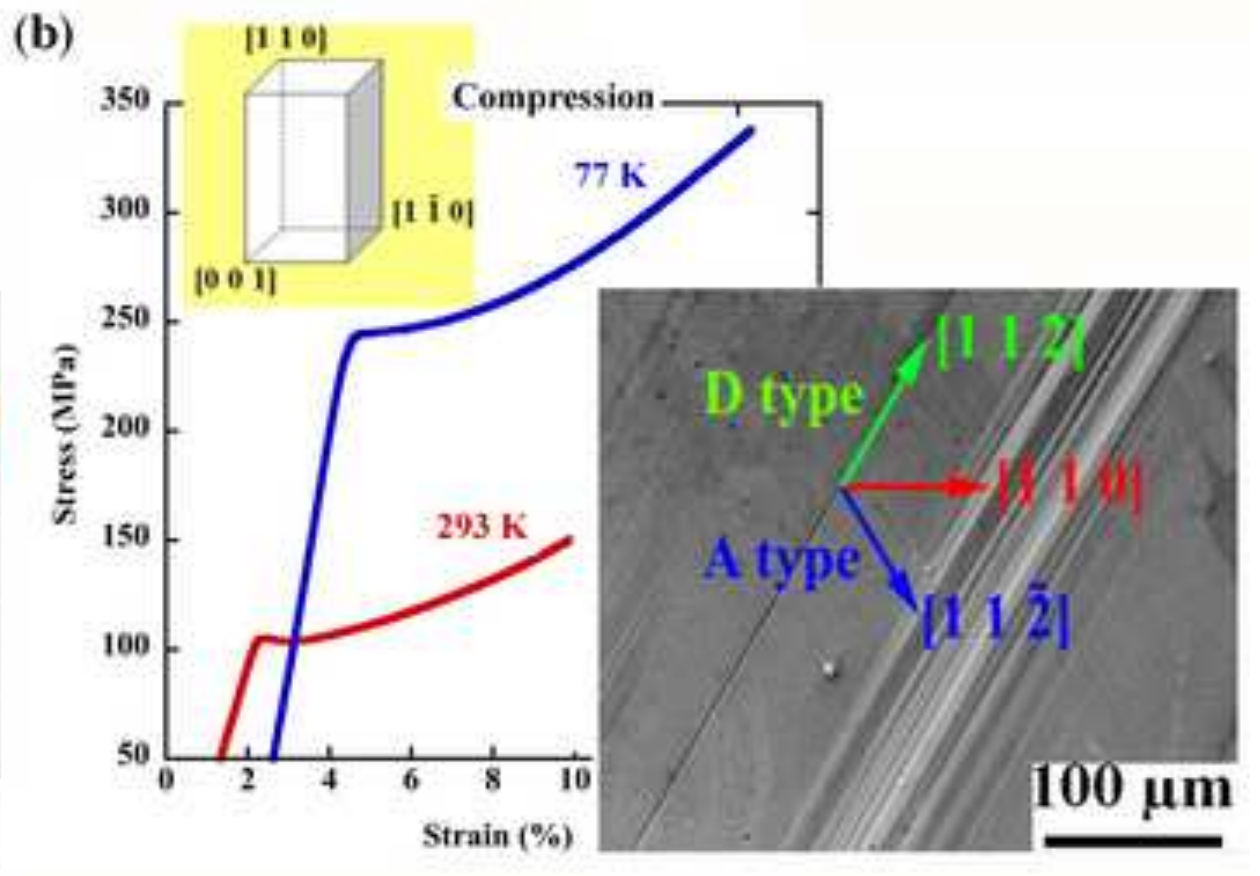


(a)

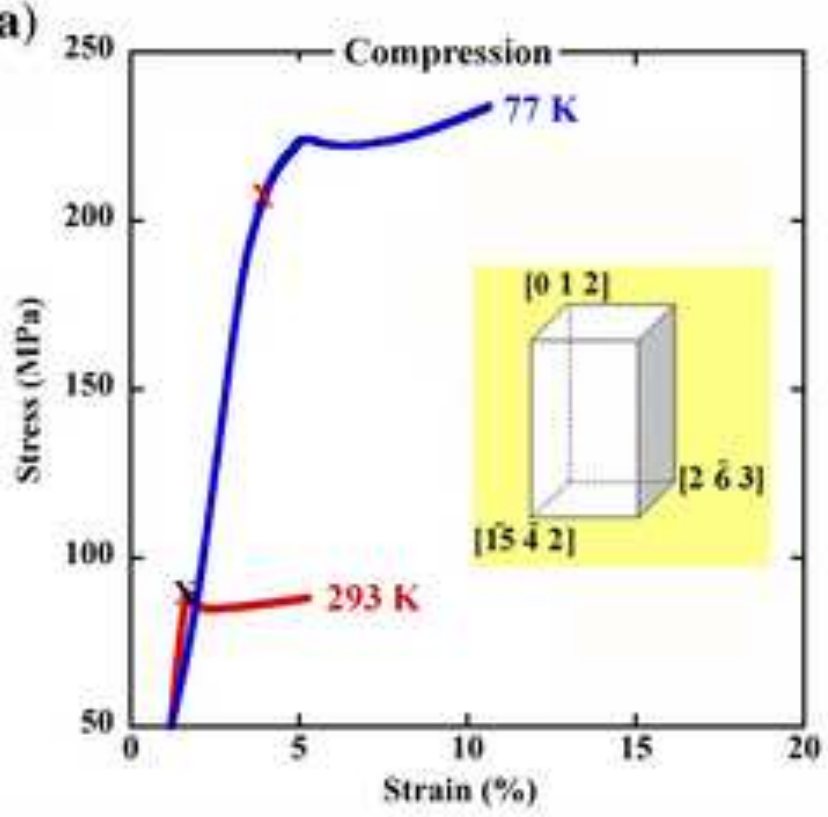

(b)

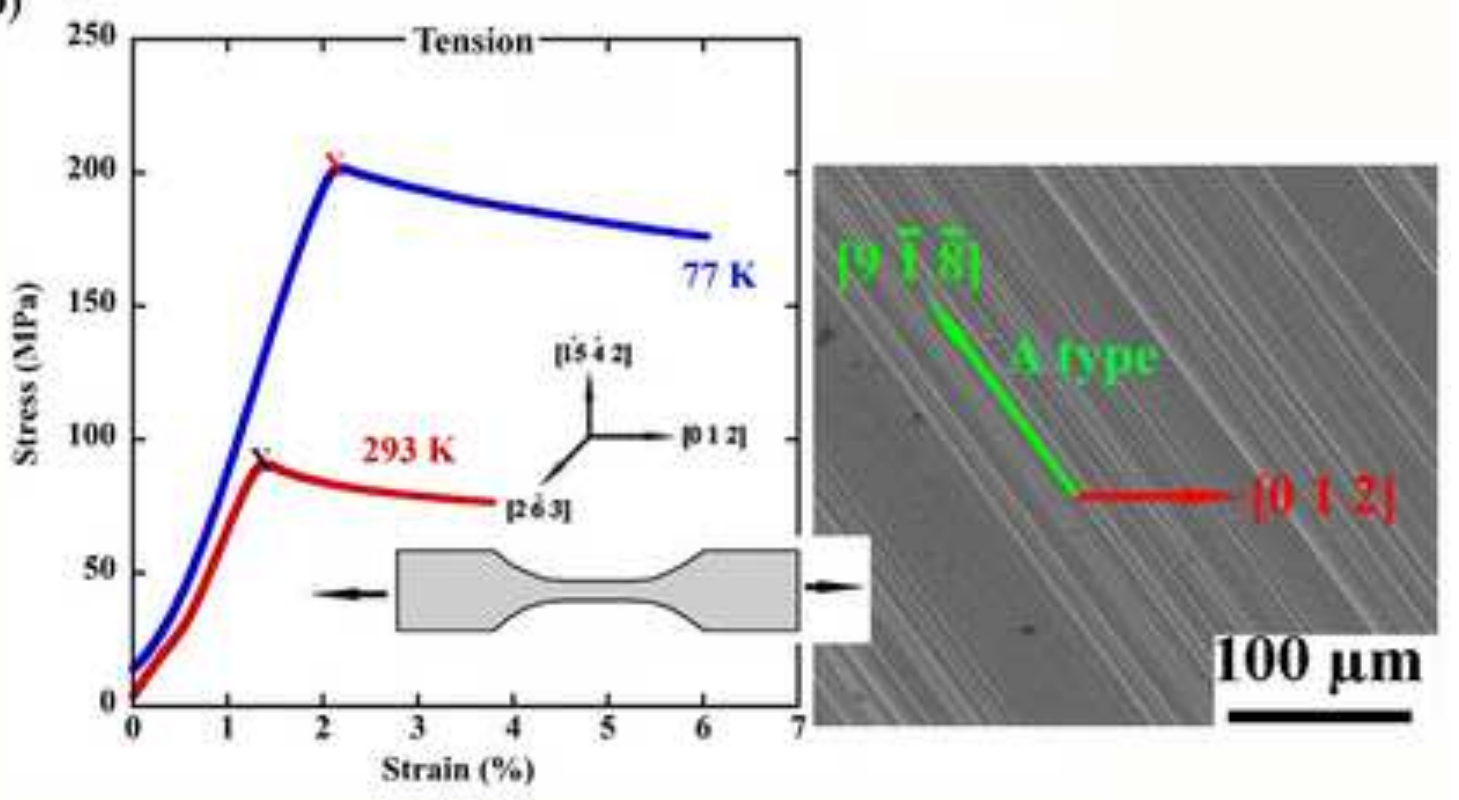

